Check for updates

Cite this: RSC Adv., 2018, 8, 26440

\title{
Magnetic response of chlorophyll self-assembly within hydrogel: a mechanistic approach towards enhanced photoharvesting $\dagger$
}

\author{
Pubali Mandal, $\ddagger^{\mathrm{a}}$ Jhimli S. Manna, (D) $\ddagger^{\star \mathrm{ab}}$ Debmallya Das, ${ }^{\text {ce }}$ Ramaprasad Maiti, ${ }^{\text {cd }}$ \\ Manoj K. Mitraae and Dipankar Chakravorty ${ }^{\dagger}$
}

Self-assembly of chlorophyll-a (Chl-a) molecules within a protein environment serves as the key factor behind controlled and efficient light energy harvesting in natural photosystems. Long-range ordering among supramolecular structures in terms of spin-orbit coupling and edge effect helps in untrapping of excitons in the disordered energy landscape. Mimicking the photosynthetic machinery would give a new paradigm for organic photovoltaic material design where a large amount of disorder exists. In this paper, we report the experimental evidence of room temperature magnetic domain wall formation and edge effect along with spin flop canting in self-assembled Chl-a within hydrogel matrix via SQUID magnetometry. This was further correlated with intermolecular coupling and exciton delocalization through specific arrangements of self-assembly as evident from NMR spectral and photophysical characteristics. The data cumulatively suggest electronic backscattering protection which is also substantiated by the ferroelectric behavior coming from coexisting symmetry lowering. Here the polarization evolves through primary distribution of $\pi$ electronic density along with a photoresponsive IV loop, similar to the photoprotection of photosynthesis. This work thus proposes a promising design principle for room temperature Chl-a based biomimetic systems efficient in photoharnessing.

Received 30th May 2018

Accepted 8th July 2018

DOI: $10.1039 / \mathrm{c} 8 \mathrm{ra0} 4612 \mathrm{c}$

rsc.li/rsc-advances
But, in vitro, most of the porphyrin-based systems suffer from Anderson localization effect of the single-particle exciton eigenstates, which reduces energy transport significantly. YuenZhou et al. ${ }^{7}$ reported that topological Frenkel exciton edge states in 2D porphyrins can be robust against disorder through symmetry constraint. We aimed to mimic this idea of nature's topologically protected antenna complex containing asymmetrical chromophores with strong spin-orbit-coupling edges through experiments. ${ }^{7-9}$ We studied interactions among Chla in polyacrylamide hydrogel matrix (PaaChl) at room temperature via SQUID, NMR and photophysical properties to investigate the possibility of excitons being untrapped through disorder, edge effects and spin-orbit coupling. The broader aspect is to focus on the underlying mechanism for good photocurrent efficiency. Therefore this work offers a novel approach to elucidate design principles for synthetic structural scaffolds or matrices, towards an efficient light harvesting scheme. Also, natural pigments like Chl-a are attractive photosensitizers because they are of low cost, abundant in supply, and sustainable.

\section{Experimental}

\section{Synthesis procedure}

The synthesis procedure was the same as described in our previous publication. $^{{ }^{10}}$ Acrylamide and $N, N^{\prime}$ - 
methylenebisacrylamide were mixed in DI water and stirred for 30 minutes. The mixture was kept under vacuum for 30 minutes, followed by addition of ammonium persulfate solution and tetramethylethylenediamine. ${ }^{11,12}$ The $\mathrm{pH}$ of the solution was measured to be $\sim 6.5$. This solution was kept under vacuum for 2 days for drying. The as-prepared hydrogel was washed in water several times, wiped thoroughly, and was soaked in Chl-a overnight followed by removal of surface pigments through washing. For light-induced $J-V$ measurements, PaaChl was sandwiched between two electrodes (ITOcoated glass as the transparent working electrode on top, Agcoated substrate as the counter electrode) (Fig. S1C and $\mathrm{S} 1 \mathrm{D} \dagger$ ) having active area of $0.64 \mathrm{~cm}^{2}$ and thickness of $1 \mathrm{~mm}$.

\section{Materials characterization}

UV-visible absorption spectra were measured using a Lambda 35 UV-visible spectrometer (PerkinElmer). Time correlated fluorescence decay data were collected with a Hamamatsu MCP photomultiplier (R3809) and were analyzed using IBH DAS6 software. Fluorescence anisotropy measurements were carried out with a Horiba Jobin Yvon IBH, JY-IBH 5000M set. Fourier transformed infrared spectra were measured using an IR Prestige 21 (Shimadzu) spectrometer (resolution $4 \mathrm{~cm}^{-1}$ ). Raman scattering spectra were obtained using a Trivista 555 spectrograph of Princeton Instruments at an excitation wavelength of $413.1 \mathrm{~nm}$ from a $\mathrm{Kr}$ ion laser. The resolution of the resonance Raman instrument was $0.5 \mathrm{~cm}^{-1}$, with grating number $900 / \mathrm{mm}$. ${ }^{1} \mathrm{H}$ NMR spectra were measured using a Bruker Ascend $600 \mathrm{MHz}$ set up. Magnetic property measurements were carried out with a Quantum Design MPMS system having a SQUID magnetometer in the temperature range $2-300 \mathrm{~K}$. The magnetization values were measured under zero field-cooled (ZFC) and fieldcooled (FC) conditions at an applied magnetic field of 100 Oe. Photocurrent responses were measured with a Keithley 238 source meter under white light illumination of $1000 \mathrm{~W} \mathrm{~cm}^{-2}$ power at room temperature.

\section{Results and discussion}

\section{Photophysical property analysis}

We earlier designed the PaaChl system via two different procedures: swelling induced and in situ, where the former results in greater photocurrent; however all other parameters remain constant. ${ }^{10}$ This phenomenon was further correlated with the high degree of spatial arrangement of stacked Chla molecules within gel matrix, as evident from UV-visible and time-dependent spectroscopic study. Steady-state absorption spectrum [Fig. 1A] clearly shows the presence of liquid crystalline like structure which is randomly oriented in the in situ sample. ${ }^{13}$ The degree of randomness of these ordered molecular stacks throughout the gel is much higher, as evident from fluorescence anisotropy measurement [Fig. 1C and D] where the decay curve is random and could not be fitted to any particular function. This can arise due to the presence of multiple excited states, each following different depolarization processes, arranged in a complete inhomogeneous manner throughout
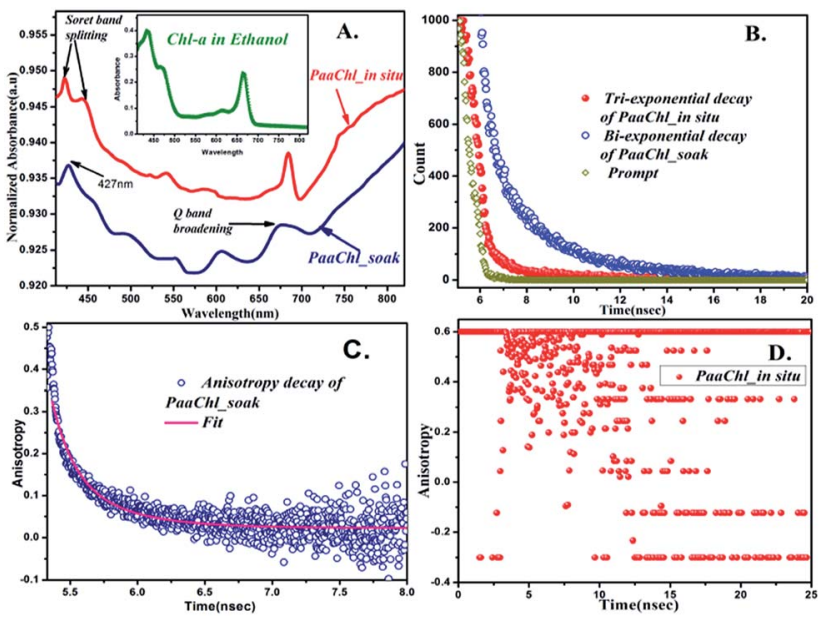

Fig. 1 Optical spectroscopy data of PaaChl (swollen and in situ; reprinted with permission). (A) UV-visible absorption spectra. (B) TCSPC data. (C) Fluorescence anisotropy decay of PaaChl_soak. (D) Fluorescence anisotropy decay of PaaChl_in situ.

the matrix. The swollen counterpart showed high initial anisotropy as a signature of arranged excitonically coupled packet towards excitonic decay supporting the evidence of longrange ordering [UV-visible spectra, Fig. 1A] within gel matrix. The shorter component [ 55 ps, Fig. 1C] of bi-exponentially fitted fluorescence anisotropy decay can be related to the fate of the exciton where excitonic packets break the symmetry. The exciton may reside in the trap state and find a non-radiative path to dissipate or emit photon as luminescence through radiative decay. The evidence of long-range ordering in anisotropy and steady-state absorption spectra [Fig. 1A, up to $800 \mathrm{~nm}$ ] points to the fact that the excitonic packets are coupled within the swollen sample. Thus non-radiative decay cannot be inferred as responsible for reducing energy transport as we found larger photocurrent within this sample.

Time-correlated single-photon counting (TCSPC) data also showed an even population distribution of two different supramolecular species in swollen sample [Fig. 1B] with 2.962 ns and 0.868 ns timescale, which cannot be related to the presence of dimer or monomer. We also did not find any significant signature of monomer in UV-visible spectra [Fig. 1A]. With these evidences it can be inferred that some of the excitons become robust against disorder giving rise to higher photocurrent density stemming from the high degree of spatial arrangement (as this is the only difference found among the two samples). This is similar to photosynthetic antenna complexes where chlorophylls are in a specific arrangement in the protein matrix giving rise to the maximum photoconversion efficiency, exemplifying the properties of topological protection at ambient temperature. Our polyacrylamide scaffold plays a similar role by introducing a dielectric quasi-liquid environment to modify electronic coupling among three-dimensional pigment arrangements. Interestingly, we found photo IV loop similar to photoprotection during photosynthesis [Fig. 7B, inset] which supports the presence of topological protection. The excited 
states follow different pathways for forward and backward excitonic movements in the absence of any back reflections at an arbitrary abruption or discontinuity. ${ }^{14-16}$

\section{Magnetic data analysis}

In order to investigate magnetic response regarding topological protection we performed SQUID magnetometry of the swollen sample only to come across very interesting results in regard to magnetic susceptibility. The isothermal magnetization data [Fig. 2A] at $300 \mathrm{~K}$ could be satisfactorily fitted with a Langevin function $L(x)$ valid for classical spin of $S=\frac{1}{2}$, corresponding to disordered magnetic behaviour. A magnetic moment [after fitting Fig. 2C] of $44.7 \mu \mathrm{B}$ (ref. 17) suggests a clustering of the $S=$ $\frac{1}{2}$ spins in the porphyrin assembly. The low-temperature region deviates from the Langevin function due to interparticle interactions, ${ }^{18}$ anisotropy ${ }^{17}$ and in homogeneities such as volume and moment distribution. In the temperature range of 2-120 K the measurement shows a large difference between ZFC and FC indicating irreversibility, with no bifurcation temperature.

The ZFC curve [Fig. 3] shows a broad maxima ( $\left.T_{\max }\right)$ at temperature starting from $120 \mathrm{~K}$, featuring a behaviour similar to commonly known metastable magnetic systems like spin glasses, cluster glasses, super-paramagnets or randomly canted ferromagnets. We exclude the existence of all these systems other than randomly canted ferromagnets, because of the fact that we found $T_{\max }$ with no bifurcation temperature which may reside in a much higher regime (above $300 \mathrm{~K}$ ). The FC and ZFC magnetization becomes stable without saturation above $T_{\max }$, also hinting at the presence of randomly canted ferromagnets. The existence of maxima in ZFC data can be a consequence of competing interactions and/or low dimensionality of the magnetic system. $T_{\max }$ marks a crossover region where the average anisotropy energy and the thermal energy $\left(K_{\mathrm{B}} T\right)$ are
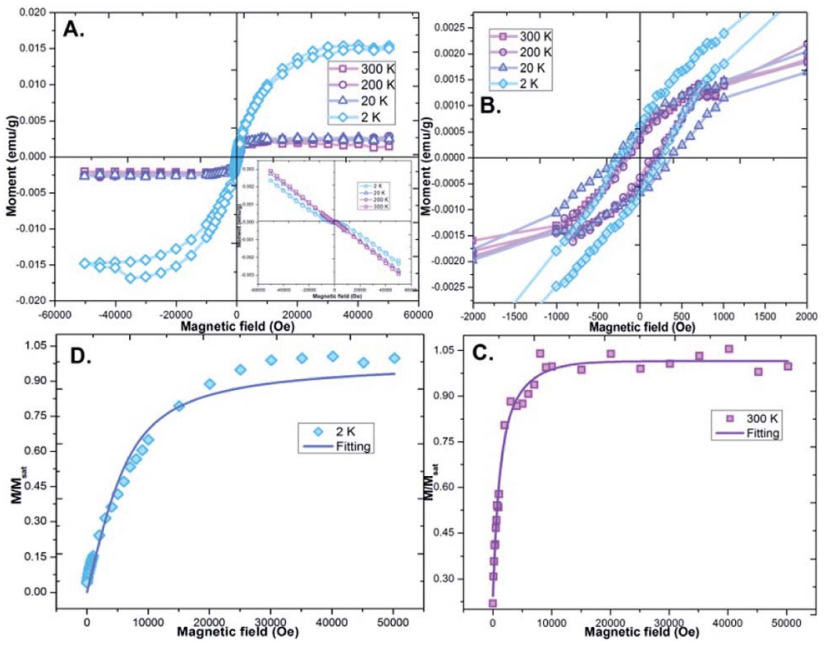

Fig. 2 Magnetic data. (A) Isothermal $M-H$ loops obtained at different temperatures between 300 and $2 \mathrm{~K}$. Inset shows magnetic hysteresis data without diamagnetic correction. (B) Closer view of the low magnetic field regions of the $M-H$ loops. (C and D) Langevin fitting of $300 \mathrm{~K}$ and $2 \mathrm{KM}-\mathrm{H}$ curves respectively.

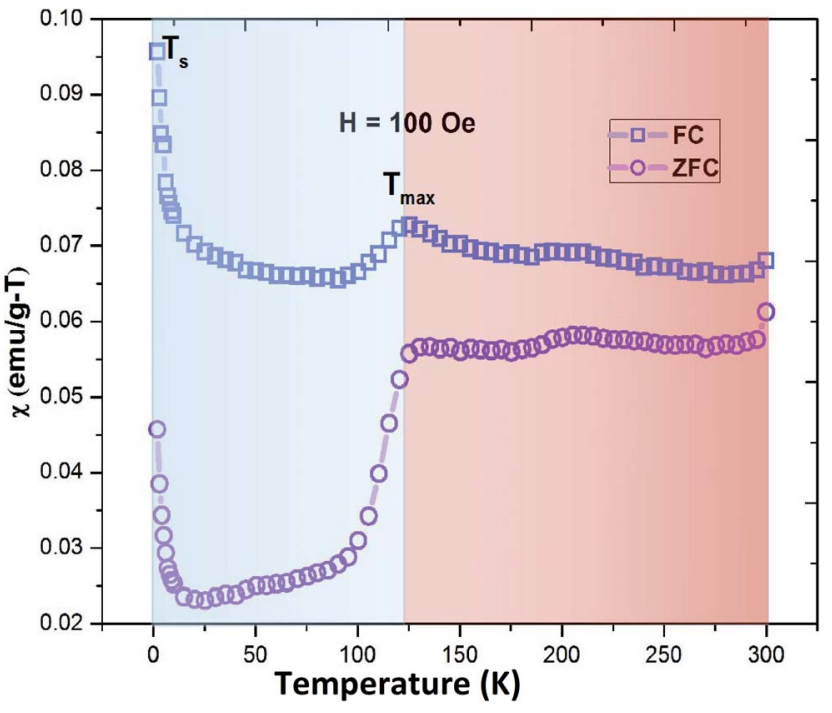

Fig. 3 Temperature-dependent magnetic study. ZFC and FC measurement curves of PaaChl at 100 Oe magnetic field in the temperature range $300 \mathrm{~K}$ to $2 \mathrm{~K}$.

comparable and the thermal energy is predominant for $T>T_{\max }$. The observed behaviour reveals progressive magnetic moment blocking, with a distribution of relaxation times related to the size and anisotropy axis direction distributions. The lowtemperature sharp maximum in MT curve $[T=125 \mathrm{~K}$, Fig. 3] can be correlated to the existence of magnetically disordered states within a diamagnetic matrix and points out the competing interactions among the different Chl-a assemblies. At temperature $T=15 \mathrm{~K}$ magnetization reversal occurs locally because strongly pinned domains change orientation without propagation of domain walls. At low temperature, competing magnetic interactions stabilize a spin-glass-like state at the interface, yielding freezing of those surface spins in random directions. This, in turn, inhibits the exchange interaction to transmit across the interfaces, resulting in uncoupling of each Chl-a domain similar to perovskite material where symmetry breaking is crucial. The broad maximum of ZFC above $T_{\max }$ is associated with the blocking of magnetically aligned self-assembled Chla moments which change orientation at $T=15 \mathrm{~K}$. The sharp maximum at $T_{\mathrm{S}}$ is related to the freezing of surface spins. The FC curve does not superimpose the ZFC curve at temperatures above $T_{\mathrm{S}}$ because the component associated with this behaviour has an additional background due to the FC magnetization of the blocking at higher temperature. This also indicates evidence of geometric frustration as ZFC and FC largely differ below $T_{\max }$. Between $2 \mathrm{~K}$ and $30 \mathrm{~K}$ [Fig. 2], the magnetization curves of PaaChl reveal hysteresis with non-zero coercivity. A change of hysteretic loop shape is observed below and in the vicinity of $T=2-30 \mathrm{~K}$ [Fig. 2A and B], characterized by narrowing of the cycle in the lowtemperature regime without saturation of the magnetization. This supports the already proposed mechanism that at low temperature magnetically disordered surface frozen spins are responsible for the change in the shape of the hysteresis loop at $T$ close to $T_{\mathrm{S}}$. The above $T_{\max }$ hysteresis behaviour corresponds to the growing fraction of blocked magnetic moments. 


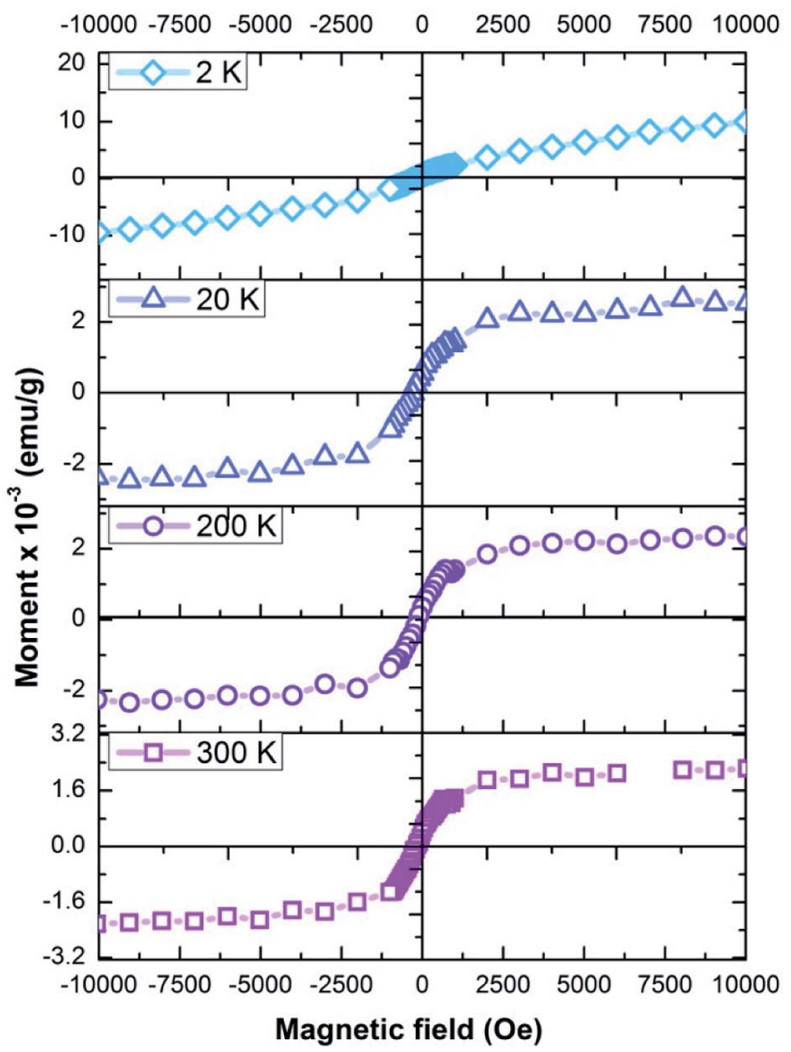

Fig. 4 Temperature-dependent magnetic study. SQUID MH measurement curves of PaaChl at 100 Oe magnetic field at temperatures of $300 \mathrm{~K}$ to $2 \mathrm{~K}$, showing squareness feature of $\mathrm{MH}$ curve.

It can be clearly seen from Fig. 4 that the squareness of the loops reduces gradually with decreasing temperature which is a direct sign of ineffective inter-domain interaction. No exchange bias phenomenon has been observed in this system due to the fact that, even if any spin flop canting can be present at the interface providing a small net magnetic moment to which the ferromagnet can couple, during magnetization reversal, a canted antiferromagnetic interface is unstable to out of plane fluctuations and can nucleate a reversal of the antiferromagnet domain producing coercivity but no shifted hysteresis loop for the ferromagnet. This domain wall formation also helps in protecting excitons and prevents electron backscattering very similar to photosynthetic systems where the excitons are topologically protected. ${ }^{19}$ Thus the magnetic behaviour arises from the cumulative contribution of Chla inter-domain interaction resulting in magnetically disordered surface frozen spins, which does not saturate even up to a field of $50 \mathrm{kOe}$, and matrix interface Chl-a interaction which tends to saturate at low fields. This system thus has the potential to carry time reversal symmetry with delocalized current carrying chiral edge modes through exciton generation over self-assembled Chl-a domain related to spin-orbit coupling which borrows strong oscillator strength in the absorption spectra ${ }^{20}$ [Fig. 1A]. Yuen-Zhou et al. ${ }^{7,21}$ proposed that ring-like porphyrin supports two degenerate excitons corresponding to clockwise or counter-clockwise electron orbital angular momentum within each ring. In the presence of a magnetic field, these states are Zeeman split in energy (as observed from NMR), as one of them is favoured and the other not favoured by the Lorentz force. The motion of these chiral excitons is governed, to a good approximation, by classical dipolar interactions. Together, the anisotropy of these interactions and the Zeeman effect produce an effect where, as the excitons move in a closed loop, they acquire a phase, in very much the same way as when electrons move in cyclotron orbits in the presence of a magnetic field. ${ }^{21}$ Topological protection is one of the prerequisites of this proposal which is related to nonrandom arrangement of molecules which is effectively evident here and which can protect the exciton to become robust. We intend to find out whether symmetry breaking related to spinorbit coupling is behind this metamagnetic phenomenon of the system through NMR spectral analysis.

\section{Infrared spectroscopy analysis}

FTIR and Raman spectra were studied for further understanding the nature of the Chl-a molecular interactions within the hydrogel [Fig. 5]. The IR peak around $900 \mathrm{~cm}^{-1}$ arises from superposition of alkene and aromatic $\mathrm{sp}^{2} \mathrm{C}-\mathrm{H}$ bending which signifies the presence of Chl-a within the hydrogel [Fig. 5A]. A small peak at $1485 \mathrm{~cm}^{-1}$ arises due to 16-membered porphyrin ring vibration. ${ }^{22}$ Blue-shifted pyrrole ring vibration at $1330 \mathrm{~cm}^{-1}$ in the Raman spectrum is a signature of strong intermolecular interaction. ${ }^{22,23}$ The Raman line at $1136 \mathrm{~cm}^{-1}$ can be related to $\mathrm{C}_{\alpha}-\mathrm{N}$ stretching. No significant change of the $\mathrm{C}=\mathrm{O}$ vibration (appearing at $1695 \mathrm{~cm}^{-1}$ for polar solvents) rules out the probability of $\mathrm{C}=\mathrm{O} \cdots \mathrm{H}_{2} \mathrm{O} \cdots \mathrm{Mg}$ or $\mathrm{C}=\mathrm{O} \cdots \mathrm{Mg}$ pair formation, whereas Raman lines at $1634 \mathrm{~cm}^{-1}$ and $1672 \mathrm{~cm}^{-1}$ clearly corroborate that the central $\mathrm{Mg}$ is 5 -coordinated which is further related to porphyrin non-planarity. ${ }^{24}$ Strong intermolecular coupling between closely packed porphyrins is facilitated in the water-rich dielectric environment of hydrogel matrix. This results in redistribution of energetic landscape through pigment-matrix and pigment-pigment interactions (as supported by IR and Raman data) which is reflected in redshifted broadened $\mathrm{Q}$ band at $673 \mathrm{~nm}^{25,26}$ [Fig. 1A]. Two broad humps around $550 \mathrm{~nm}$ (Soret band) and $612 \mathrm{~nm}$ (Q band) can be correlated to the perturbation of Chl-a planarity (further supported by NMR) assisted via the presence of various matrixporphyrin interactions (conjugated carbonyl group, hydrogen bond, $\mathrm{Mg}$ 5-coordination, as supported by Raman and NMR later) which distinguishes between $x$ and $y$ directions of electronic transition, giving rise to the spin-orbit coupling suggesting that the multiplicity-forbidden transition is realized through intermolecular interaction of chiral Chl-a assembly. The broadening also can be attributed to an enhancement in the probability of forming an ensemble distribution of excitonically coupled conformational isomers, which results in an overall broad absorption envelope. ${ }^{10,27}$

\section{NMR spectrum analysis}

Chl-a has asymmetric centres at $\mathrm{C} 7, \mathrm{C} 8, \mathrm{C} 10, \mathrm{C}^{\prime}$ and $\mathrm{Cl}, 1^{\prime}$ with the configurations $7(S), 8(S), 10(R), 7^{\prime}(R), \mathrm{l}$ and $\mathrm{l}^{\prime}(R)$, having the 

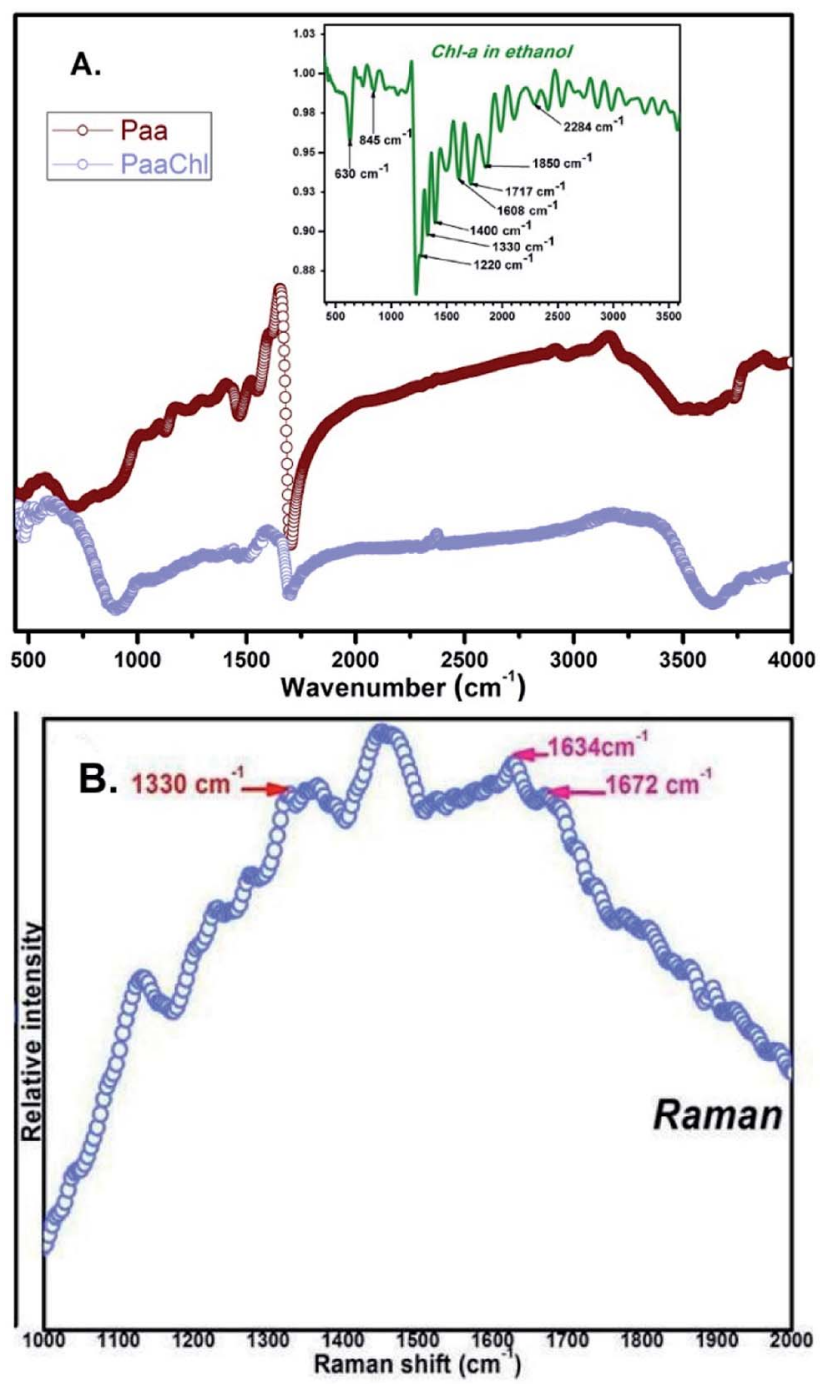

Fig. 5 Infrared spectroscopy study of PaaChl. (A) FTIR spectra. (B) Raman spectrum

potential to show spin selectivity through spin-orbit coupling when in assembled form. The downfield shift of $13^{2} \mathrm{H}$ indicates major deshielding [Fig. 6], caused by macrocycle distortion through $5^{\text {th }}$ coordination (as evident from Raman spectrum, Fig. 5B) which moves it away from the macrocycle plane. P9, P8, P5 triplet coupling and high-field shift suggest that the propionate side chain is away from the macrocycle, as the distortion is on the opposite side of the phytol chain. These alterations were attributed to the steric crowding arising between the protons of $13^{2}$-methoxycarbonyl group and the bulky C17 propionic acid phytol ester moiety. These substituents become oriented on the same side of the macrocyclic ring plane, indicating the presence of Chl-a' $=13^{2}(S)-\mathrm{Chl}-$ a [Fig. 6] resulting in a weakened electron donating property of carbonyl groups present in the lower periphery of this isomer. ${ }^{28}$ The higher-field shifts observed in most of the assignments suggest that larger aggregates were present which have a layered structure (otherwise, the complexation shifts would not be further to higher field) which is not a direct

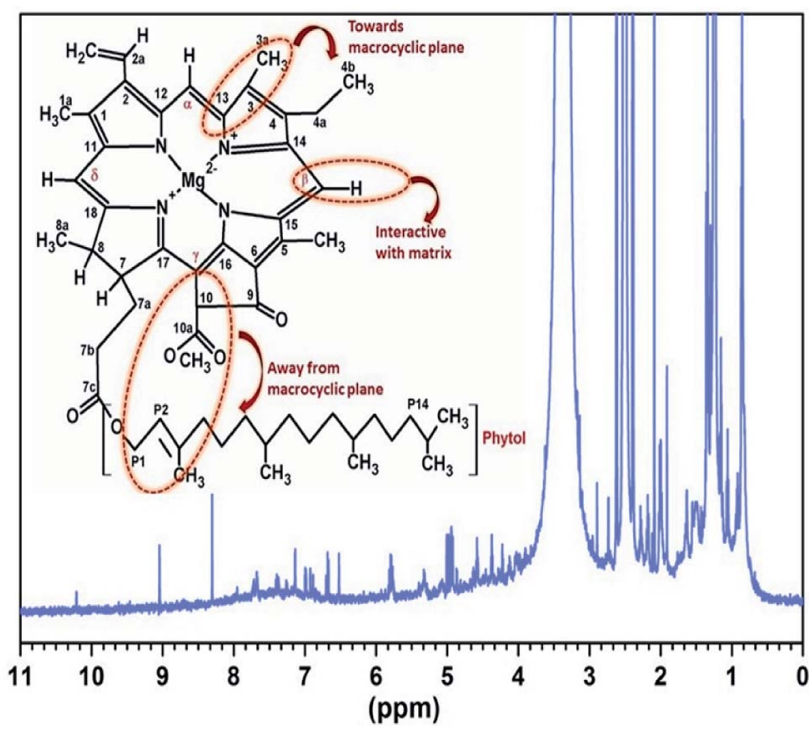

Fig. $6{ }^{1} \mathrm{H}$ NMR study of PaaChl. Inset: structure of chlorophyll-a. The highlighted areas show main regions of molecular interactions as found from NMR data analysis.

extension of the dimer structure. ${ }^{28}$ For example major upfield shift of $\alpha$ (9.29 to $9.078 \mathrm{ppm}$ ) and $\delta$ protons (8.32 to $8.28 \mathrm{ppm}$ ) when compared to monomeric Chl-a. ${ }^{29}$ These data reveal that these protons in Chl-a are in the shielding cone of the ring current and closer to the macrocycle assembly. Macrocycles come closer to these out of plane protons after complexation and aggregation through water molecules. Both $18 \mathrm{H}$ and $17 \mathrm{H}$ experience shielding and triplet splitting $(4.6 \mathrm{t} ; J=5.46,4.5 \mathrm{q} ; J$ $=2.16,11.16$ ) which may be due to adjacent methyl for $18 \mathrm{H}$ and adjacent $\mathrm{CH}_{2}$ of phytol for $17 \mathrm{H}$ simultaneously, which also supports the crowding of these groups towards the same plane through distortion of the macrocycle. The $4.97 \mathrm{~d}(J=1.56)$ and $5 \mathrm{~d}(J=1.56)$ coupling transition arises due to the interaction of $10 H \cdots \mathrm{P} 2$ which may interact with P1 [Fig. S3†]. Bending makes them move away from the macrocycle causing downfield shift and makes them come closer to the neighbouring $10 H$ carboxymethyl group, which causes the dd splitting $(7.135 \mathrm{~d} ; J=2.34,7.38 \mathrm{dd} ; J=2.7,5.94)$. Another two very close peaks at 4.94, $4.92 \mathrm{ppm}$ may come from slightly different sterically restricted conformer where P1, P2 interact. P1 bends away from shielding, implying severe distortion in planarity, especially in ring 4 and 5 through large steric interaction, and greater shift values observed for $\mathrm{Pl}-\mathrm{CH}_{2}, \mathrm{P} 2 \mathrm{H}$, and $\mathrm{P} 3 \mathrm{a}-\mathrm{CH}_{2}$ protons reveal the close proximity of this olefinic region of the phytol group and the methoxycarbonyl group. This fact also supports the presence of Chl-a $S$ form. ${ }^{28}$ Absence of $\beta$ carbon (usually at $9.54 \mathrm{ppm}$ ) may be due to the deprotonation as a result of the interaction between severely distorted Chla with the surrounding polyacrylamide- $\mathrm{NH}_{2}$ which can act as proton acceptor or organic base resulting in deprotonated Chla assembly. The steric crowding weakens the electron-pair donor properties (nucleophilicity) of the three carbonyl groups in the lower periphery and points out the presence of the chlorophyll $13^{2}(S)$-epimer, having different coordination 
Table $1{ }^{1} \mathrm{H}$ NMR chemical shifts and observed coupling constants in PaaChl

\section{NMR shift}

\begin{tabular}{lll}
$(\mathrm{ppm})$ & Assigned proton & Coupling constant $(\mathrm{Hz})$ \\
\hline 10.21 & $13^{2}$ & $10.21 \mathrm{~d}, J=0.48$ \\
9.078 & $\mathrm{~A}$ & $\mathrm{~s}$ \\
8.28 & $\Delta$ & $\mathrm{s}$ \\
7.95 & $2 \mathrm{a}$ & $7.95 \mathrm{~d}, J=1.8$ \\
7.38 & P1 bending & $7.38 \mathrm{q}, J=2.76,5.94$ \\
7.135 & $2 \mathrm{~b}$ & $7.135 \mathrm{~d}, J=2.34$ \\
6.98 & P1 bending & $6.98 \mathrm{dd}, J=2.37,8.55$ \\
5 & $10 H-\mathrm{P} 2$ interaction & $5 \mathrm{~d}, J=1.56$ \\
4.97 & $10 H-\mathrm{P} 2$ interaction & $4.97 \mathrm{~d}, J=1.56$ \\
4.94 & P1-P2 interaction & $\mathrm{s}$ \\
4.6 & $18 H$ & $4.6 \mathrm{t}, J=5.46$ \\
4.5 & $17 H$ & $4.5 \mathrm{q}, J=2.16,11.16$ \\
3.36 & $1 \mathrm{a}$ & $\mathrm{s}$ \\
2.895 & $7 \mathrm{a}$ & $\mathrm{s}$ \\
2.74 & $7 \mathrm{a}^{\prime}$ & $\mathrm{s}$ \\
2.09 & $7 \mathrm{~b}^{\prime}$ & $\mathrm{s}$ \\
1.907 & $\mathrm{P} 4$ & $\mathrm{~s}$
\end{tabular}

and hydrogen-bonding properties from $13^{2}(R)$-chlorophyll (10.21d, $J=0.48)$, (10.2d, $J=1.92)$. The $13^{2}(S)$-epimeric Chla can only be produced through the Chl-a enol intermediate, through hydrogen bonding with surrounding water-rich environment, which implies that complexation through $\beta$-keto ester group predominates in the system. This Chl-a enol intermediate is very common in the photosynthetic reaction center. ${ }^{26}$ Other proton shifts are listed in Table 1 . All these data cumulatively suggest the presence of a sterically constrained, non-planar Chl-a chiral assembly, where spin-orbit coupling due to the presence of differently oriented chiral centers gives the possibility of enantio-selection assisted by symmetry breaking. Although the specific repetition cannot be confirmed, correlating excitonic data with the NMR data we suggest that the two types of arrangement (of $S R$ conformer) are present almost in equal proportion as the population ratio of biexponential decay curve has two different lifetimes [Fig. S $3 \dagger$ ] of almost 0.5. This information points toward a specific dynamic arrangement within the hydrogel to form a basis of excitonic coherence at room temperature.

\section{Electrical measurement data}

We further extended our observations to investigate the PE loop [Fig. 7] of the PaaChl/electrode ensemble which shows a leaky ferroelectric nature resulting from asymmetric and nonlinear photocarrier dynamic ${ }^{30}$ (as evident from photoresponse measurement, Fig. 7B). This ferroelectric behavior comes from coexisting symmetry lowering via the domination of electron-lattice interactions (topological interaction also evident from SQUID) resulting in the Peierls deformation where the polarization involves primarily distribution of $\pi$ electronic density rather than displacement of ions. ${ }^{31}$ The lifted degeneracy of chiral Chl-a assembly through symmetry breaking (NMR, Fig. 6) results in firstly quasi one-dimensional
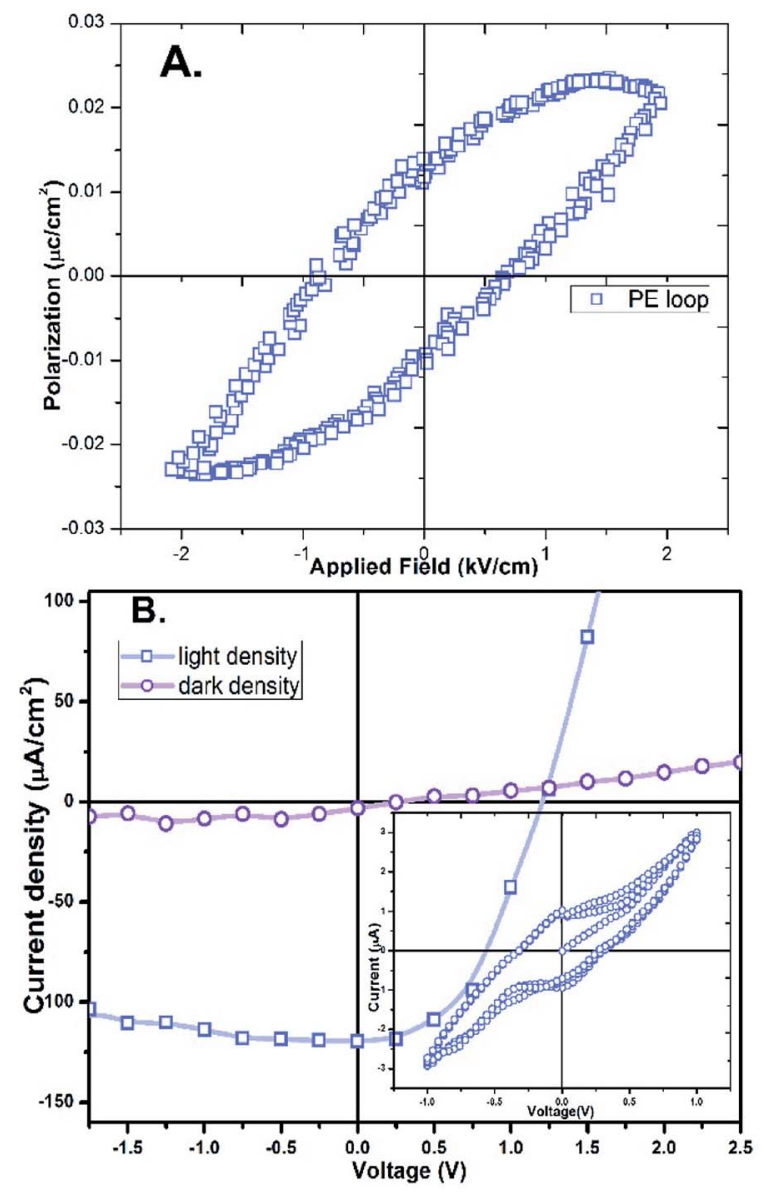

Fig. 7 Electrical measurement data. (A) Room temperature PE loop of PaaChl showing ferroelectric signature. (B) $I-V$ characteristic curves of PaaChl under dark and illuminated conditions.

Table 2 Photo $1-V$ response of PaaChl

\begin{tabular}{llll}
\hline$J_{\text {sc }}\left(\mu \mathrm{A} \mathrm{cm}^{-2}\right)$ & $V_{\text {oc }}(\mathrm{V})$ & $\mathrm{FF}$ & PCE $(\%)$ \\
\hline 120.2 & 1.199 & 0.463 & 0.667 \\
\hline
\end{tabular}

chains of Chl-a, each carrying a spin of $\frac{1}{2}$, then formation of $3 \mathrm{D}$ ordering, giving rise to macroscopic domain wall, from these excitonic aggregates of opposite spin polarization, as evident in SQUID magnetometry. This may cause areas of fluctuating electronic dipoles to couple to magnetic domains; domain walls between magnetic domains in addition to spins carry charge which accumulates within domain boundaries in order to promote the formation of magnetic order. The robustness of this spin alignment against temperature as evident from MT curve in SQUID points to the spin-phonon coupling similar to the characteristic of organic donor-acceptor type ferroelectric spin Peierls system. ${ }^{32,33}$ The FF and external PCE of the PaaChl/ electrode assembly were found to be 0.463 and $0.667 \%$ respectively [Table 2]. The bare hydrogel (Paa) does not show any significant change in current density due to light exposure [Fig. $\mathrm{S} 4 \dagger$. 


\section{Conclusions}

This work, for the first time, gives experimental evidence of room temperature magnetic domain wall formation and edge effect along with spin flop canting in self-assembled Chl-a within hydrogel matrix via SQUID magnetometry. Such room temperature ferromagnetic and ferroelectric ordering of Chl-a molecules within a matrix is rare. Natural photosystem like topological protection through domain wall formation leading to efficient photocurrent efficiency was achieved. This phenomenon has also been substantiated by the absence of any exchange bias as a result of unrelated spin flop canting. This was further correlated with intermolecular coupling and exciton delocalization through specific arrangements of self-assembly as evident from NMR and photophysical characteristics. Spin-orbit coupling, as a result of symmetry breaking in the Chl-a assembly through deformations and reorientations of the macrocycle, can be the underlying reason of this ferromagnetic ordering at room temperature. This arrangement is spontaneous and occurs through stress-induced self-assembly. The large distribution of absorption landscape of overlapping small excitonic packets is also a signature of topologically nontrivial excitonic state among the Chl-a assembly. The presence of photo IV loop just like in a photosystem signifies that non-photochemical energy quenching protection occurs. Ferroelectric behaviour arising from Peierls deformation through spin-lattice interaction was also observed. This points out that the photocurrent response may be a result of this symmetry protection through lattice interaction where the interactive edges can assist the propagation of excitons against localization. Therefore, bridging the gap between various photophysical properties, NMR data, magnetic and electric ordering phenomenon, this work offers a novel approach to elucidate design principles for bio-inspired efficient light harvesting schemes.

\section{Author information}

P. M. synthesized the material, designed and fabricated the device, analysed optical, electrical, NMR and magnetic data and helped in writing the manuscript. J. S. M. analysed all data and correlated the analysed data and wrote the entire manuscript. D. D. helped in device fabrication, analysed optical, electrical and magnetic experimental and theoretical calculations of data and assisted with aesthetic presentation. R. M. performed the SQUID and IV experiment. MKM analysed data. D. C. correlated the analysed SQUID and PE loop data. All authors discussed the results and contributed to the manuscript. All authors have given approval to the final version of the manuscript.

\section{Conflicts of interest}

There are no conflicts to declare.

\section{Acknowledgements}

We are grateful to the Central Research Facility of Indian Institute of Technology, Kharagpur for providing NMR facility.
We gratefully acknowledge Prof. Subhasish Basu Majumder of Materials Science, Indian Institute of Technology, Kharagpur who provided the electrical polarization measurement facility. The authors are grateful to Prof. S. Baitalik of Department of Chemistry, Jadavpur University for his help in TCSPC experiments and analysis. The technical assistance rendered by $\mathrm{Mr}$ Subrata Das for anisotropy characterization from Indian Association Cultivation of Science, Kolkata, India is acknowledged as well. Help rendered by Dr Pushan Banerjee of School of Energy Sciences, Jadavpur University in photocurrent measurements is also gratefully acknowledged. Financial support from UGC; UPE-II, CSIR; RA and SERB; NPDF scheme to the authors is also gratefully acknowledged.

\section{References}

1 Z. Yan, Y. Li, Y. Gao, J. Wen, L. Hu and J. You, Opt. Mater., 2017, 73, 393-399.

2 Z. Yan, S. Guang, X. Su and H. Xu, J. Phys. Chem. C, 2012, 116, 8894-8900.

3 L. Nie, Q. Zhang, L. Hu, Y. Liu and Z. Yan, Sens. Actuators, B, 2017, 245, 314-320.

4 Z. Yan, S. Guang, H. Xu, X. Su, X. Ji and X. Liu, RSC Adv., 2013, 3, 8021-8027.

5 Z. Yan, S. Guang, H. Xu and X. Y. Liu, Dyes Pigm., 2013, 99, 720-726.

6 B. R. J. P. Grimm, W. Rudiger and H. Sheer, Chlorophylls and Bacteriochlorophylls, Springer, 2006.

7 J. Yuen-zhou, S. K. Saikin and N. Y. Yao, Nat. Mater., 2014, 13, 1026-1032.

8 W. Auwärter, D. Écija, F. Klappenberger and J. V Barth, Nat. Chem., 2015, 7, 105-120.

9 G. D. Scholes, G. R. Fleming, A. Olaya-castro and R. Van Grondelle, Nat. Chem., 2011, 3, 763-774.

10 P. Mandal, J. S. Manna, D. Das and M. K. Mitra, RSC Adv., 2016, 6, 90280-90289.

11 R. E. Rivero, M. a. Molina, C. R. Rivarola and C. a. Barbero, Sens. Actuators, B, 2014, 190, 270-278.

12 J. Chen and K. Park, J. Controlled Release, 2000, 65, 73-82.

13 F. Koch, M. Kullmann, U. Selig, P. Nuernberger, D. C. G. Götz, G. Bringmann and T. Brixner, New J. Phys., 2013, 15, 025006.

14 Z. Yang, F. Gao, X. Shi, X. Lin, Z. Gao, Y. Chong and B. Zhang, Phys. Rev. Lett., 2015, 114, 1-4.

15 P. Wang, L. Lu and K. Bertoldi, Phys. Rev. Lett., 2015, 115, 15.

16 A. B. Khanikaev, R. Fleury, S. H. Mousavi and A. Alù, Nat. Commun., 2015, 6, 1-7.

17 V. Tiwari, W. K. Peters and D. M. Jonas, PNAS, 2013, 110, 1203-1208.

18 A. W. Chin, S. F. Huelga and M. B. Plenio, Philos. Trans. $R$. Soc., A, 2012, 370, 3638-3657.

19 L. F. T. H. Hsieh, H. Lin, J. Liu, W. Duan and A. Bansil, Nat. Commun., 2012, 3, 1-6.

20 E. Roduner, Nanoscopic Materials: Size-dependent Phenomena, RSC, 2006.

21 J. Yuen-Zhou, SPIE Newsl., 2014, 2-4. 
22 Y. Koyama, Y. Umemoto, A. Akamatsu, K. Uehara and M. Tanaka, J. Mol. Struct., 1986, 146, 273-287.

23 J. W. Weigl and R. Livingston, J. Am. Chem. Soc., 1953, 75, 2173-2176.

24 K. Masayuki, Y. Tetsuro, H. Hidefumi and T. Hiroaki, Chem. Phys. Lett., 1990, 169, 85-88.

25 M. A. Jalaja and S. Dutta, Adv. Mater. Lett., 2015, 6, 568-584.

26 S. Vasil, S. Wiebe and D. Bruce, Biochim. Biophys. Acta, 1998, 1363, 147-156.

27 S. Kaligotla, S. Doyle, D. M. Niedzwiedzki, S. Katsumura and H. A. Frank, Photosynth. Res., 2010, 103, 167-174.

28 M. O. Senge, A. A. Ryan, K. A. Letchford, S. A. Macgowan and T. Mielke, Symmetry, 2014, 6, 781-843.
29 A. J. Raymond and A. E. Rowan, in Spectroscopy of Chlorophylls and Chlorophyll Proteins, Wiley, pp. 797-834.

30 W. Lee, J. H. Sung, K. Chu, X. Moya, D. Lee, C. Kim, N. D. Mathur, S. Cheong, C. Yang and M. Jo, Adv. Mater., 2012, 24, 49-53.

31 S. Horiuchi, Y. Tokunaga, G. Giovannetti, S. Picozzi, H. Itoh, R. Shimano, R. Kumai and Y. Tokura, Nature, 2010, 463, 789792.

32 F. Kagawa, S. Horiuchi, M. Tokunaga, J. Fujioka and Y. Tokura, Nat. Phys., 2010, 6, 169-172.

33 P. Lunkenheimer, J. Müller, S. Krohns, F. Schrettle, A. Loidl, B. Hartmann, R. Rommel, M. De Souza, C. Hotta, J. A. Schlueter and M. Lang, Nat. Mater., 2012, 11, 755-758. 